\title{
Correction to: Lineament mapping for a part of the central Sulaiman fold-Thrust Belt (SFTB), Pakistan
}

\author{
Ali Abbas Wajid ${ }^{1} \cdot$ Muhammad Anees ${ }^{2} \cdot$ Shams ul Alam $^{3} \cdot$ Jodat Kamran Gorchani $^{3} \cdot$ Khurram Shahzad $^{3} \cdot$ Asif Israr $^{3}$. \\ Muhammad Shafique $^{3}$
}

Published online: 13 September 2021

(C) Saudi Society for Geosciences 2021

\section{Arabian Journal of Geosciences (2021) 14:1438 \\ https://doi.org/10.1007/s12517-021-07784-y}

The original version of this paper was published with error. Tables 1 and 7 were published identical. Given in this article is the correct Table 7 .

Table 7 PCA eigenvalue plot showing the $\mathrm{PC} 1$ and $\mathrm{PC} 2$ axes with the highest variance

\begin{tabular}{lll}
\hline & Field joint dataset & Lineament dataset \\
\hline \% variance PC1 & 60.882 & 52.4 \\
\% variance PC2 & 27.095 & 40.66 \\
Total variance in PC (1-2) & 87.97 & 93.06 \\
PC1 Eigenvalue & 3.65 & 2.09 \\
PC2 Eigenvalue & 1.62 & 1.62 \\
Dominant factor & Joint dip & Lineament length \\
\hline
\end{tabular}

The original article has been corrected.

The online version of the original article can be found at https://doi.org/ 10.1007/s12517-021-07784-y

Muhammad Anees

muhammad.anees@stud.uni-goettingen.de

1 Institute of Applied Geosciences, Technische Universität Darmstadt, Darmstadt, Germany

2 Department for Structural Geology \& Geodynamics, GeoscienceCentre, Georg-August-University, Goldschmidtstrasse 3, 37077 Göttingen, Germany

3 Oil and Gas Development Company Limited (OGDCL), Islamabad, Pakistan 